\title{
COPP-ABVD Regimen
}

National Cancer Institute

\section{Source}

National Cancer Institute. COPP-ABVD Regimen. NCI Thesaurus. Code C64795.

A regimen consisting of cyclophosphamide, vincristine, procarbazine and prednisone

(COOP) alternating with doxorubicin, bleomycin, vinblastine and dacarbazine (ABVD), used for the treatment of Hodgkin lymphoma. 